\title{
Efeitos dos atributos do processo de mudança nas atitudes e nos comportamentos dos trabalhadores: um estudo longitudinal em uma organização pública
}

Vanessa Nery. ELETRONORTE

Elaine Rabelo Neiva. Universidade de Brasília

\section{Resumo}

Poucos estudos investigam atributos da mudança organizacional (características do contexto de mudança) que influenciam as atitudes e os comportamentos dos indivíduos. Buscando contribuir para uma maior estruturação do campo de estudos, este artigo apresenta o teste de um modelo mediacional das atitudes frente às mudanças organizacionais na relação entre atributos de mudança e os comportamentos de apoio e resistência à mudança. A análise do efeito moderador do tempo por meio de equações estruturais foi realizada por uma pesquisa com recorte longitudinal em uma organização pública brasileira. O modelo de investigação obteve bons índices de ajuste. A mediação das atitudes frente à mudança organizacional foi parcialmente corroborada e o efeito moderador do tempo foi refutado. O planejamento da mudança organizacional influencia as cognições e afetos dos indivíduos, favorecendo os comportamentos positivos de apoio à mudança. A contribuição prática se refere a um aumento da compreensão dos fatores que facilitam o sucesso das mudanças organizacionais, ressaltando que ações antecipadas e preparo dos indivíduos podem ser fundamentais.

Palavras-chave: atitudes, comportamento de apoio, resistência, mudança organizacional.

\begin{abstract}
Effects of the attributes of the process of change on workers' attitudes and behaviors: A longitudinal study in a public organization. Few studies that investigate attributes of organizational change (characteristics of the change context) that influence individuals' attitudes and behaviors. Seeking to contribute to a better structuring of the field, the present article shows a test of mediational model of attitudes towards organizational changes in relation between attributes of change and the behaviors of support and resistance to change. Considering that there are few national longitudinal studies in this area, the moderating effect of time was analyzed through structural equations. For this, a research with longitudinal waves was carried out in a Brazilian public organization. The research model obtained good adjustment indexes. The mediation of attitudes towards organizational change was partially corroborated and the moderating effect of time was refuted. The results show action planning in organizational change affects cognitions and affections of individuals, which favors positive behaviors supporting change process. The practical contribution refers to an increase in understanding which factors facilitate the success of organizational changes processes, emphasizing that anticipated actions and preparation of individuals can be fundamental.
\end{abstract}

Keywords: attitudes, change-supportive behavior, resistance, organizational change.

\section{Resumen}

Efectos de los atributos del proceso de cambio en las actitudes y los comportamientos de los trabajadores: Un estudio longitudinal en una organización pública. Pocos estudios que investigan atributos del cambio organizacional (características del contexto de cambio) que influencian las actitudes y los comportamientos de los individuos. En el presente artículo se presenta la prueba de un modelo mediático de las actitudes frente a los cambios organizacionales en la relación entre atributos de cambio y los comportamientos de apoyo y resistencia al cambio, buscando contribuir a una mayor estructuración del campo de estudios. Considerando que existen pocos estudios longitudinales nacionales en esa área, se analizó el efecto moderador del tiempo a través de ecuaciones estructurales. Para ello se realizó una investigación con recorte longitudinal en una organización pública brasileña. El modelo de investigación obtuvo buenos índices de ajuste. La mediación de las actitudes frente al cambio organizacional fue parcialmente corroborada y el efecto moderador del tiempo fue refutado. Los resultados muestran que la planificación de acciones en el cambio organizacional afecta las cogniciones y afectos de los individuos, lo que favorece los comportamientos positivos de apoyo al proceso de cambio. La contribución práctica se refiere a un aumento de la comprensión acerca de los factores que facilitan el éxito de los cambios organizacionales, resaltando que las acciones anticipadas y la preparación de los individuos pueden ser fundamentales.

Palabras clave: actitudes, el comportamiento de apoyo, la resistencia, el cambio organizacional. 
Os aspectos comportamentais e afetivos são fundamentais no tema mudança organizacional devido ao reconhecimento da importância das reações dos empregados para o sucesso das intervenções (Bordia, Restubog, Jimmieson, \& Irmer, 2011; Fugate, Prussia, \& Kinicki, 2012; Oreg, Vakola, \& Armenakis, 2011). Assim, a capacidade da organização em implementar mudanças emerge como um diferencial competitivo (Van de Ven, $\&$ Sun, 2011) e as empresas exitosas são hábeis em prever a mudança e desenvolver antecipadamente as suas estratégias (Neiva \& Paz, 2012; Van de Ven, \& Sun, 2011).

As reações dos indivíduos ao processo de mudança se tornou interesse recorrente dos estudos sobre o tema (Vakola, 2016). Existe pouco respaldo empírico na literatura sobre quais fatores influenciam os comportamentos de apoio e resistência à mudança organizacional (Kim, Hornung, \& Rousseau, 2011; Vakola, 2016), ou à forma como tais comportamentos são influenciados pelas cognições e afetos dos indivíduos (Vakola, Armenakis, \& Oreg, 2013).

As características do contexto são um importante antecedente de reações explícitas à mudança organizacional (Nery \& Neiva, 2015; Neiva \& Paz, 2012; Oreg et al., 2011), que podem ser expressas positivamente ou negativamente, e possuem componentes cognitivos, afetivos e comportamentais. Partindo do pressuposto que as atitudes frente às mudanças são tidas como reação avaliativa e afetiva, positiva ou negativa, em direção aos eventos e ao processo que ocorre na organização, e que as atitudes possuem impactos nos comportamentos humanos, é possível indicar que há uma relação com o comportamento dos indivíduos inseridos em contextos específicos de mudança organizacional (Bryson, Barth, \& Dale-Olsen, 2013, Dahl, 2011). Tais atitudes funcionam como preditores de comportamentos posteriores - de participação ou resistência - no processo de mudança (Vakola, 2016). Ao se considerar serem poucos os estudos longitudinais sobre o tema mudança organizacional (Barends, Jassen, ten Have, \& ten Have, 2014; Neiva \& Paz, 2012), não se pode assegurar que possíveis efeitos dos atributos das mudanças nas atitudes e nos comportamentos sejam estáveis ao longo do tempo (Kim et al., 2011; Vakola, 2016). Isso justifica a realização de novos estudos longitudinais, na busca de outras evidências sobre esses efeitos.

Ao se ter em conta o exposto, os objetivos do estudo foram: (a) testar a relação de mediação das atitudes frente à mudança entre variáveis atributos da mudança e os comportamentos de apoio à mudança organizacional e resistência em três momentos de coleta de dados em uma empresa pública que passava por um processo de mudança; e (b) testar se há alteração nas relações estabelecidas pelo modelo considerando três momentos de coleta e, dessa forma, analisar o efeito moderador do tempo por meio dos índices de ajuste do modelo em T1, T2 e T3.

\section{Atributos da Mudança Organizacional}

Há um esforço para mapear as características centrais que caracterizam a mudança organizacional (Bartunek \& Woodman, 2015; By, 2005). Contudo, a análise da mudança restrita à sua característica principal não oferece elementos suficientes para uma melhor compreensão do fenômeno, sendo necessário especificar seus atributos (Maes \& Hootergem, 2011). É recente a adoção do termo "atributos da mudança organizacional" como um conjunto de variáveis que caracterizam a mudança a ser investigada, portanto ainda sem consenso na literatura (Bartunek \& Woodman, 2015; Maes \& Hootergem, 2011; Neiva \& Paz, 2012).

Dentre os atributos que descrevem mudança organizacional estão: (a) controle - se refere à mudança emergente ou planejada; (b) escopo - se refere ao contínuo da adaptação à transformação; (c) frequência - se refere à quantidade mudanças organizacionais que estão acontecendo; (d) progresso - a quantidade de estágios para se implantar a mudança; (e) tempo - é a duração para se implantar a mudança; (f) velocidade - se refere ao ritmo de sucessão das ações de mudança; $(\mathrm{g})$ objetivos - refere-se ao estado final, quando a mudança é realizada; (h) estilo de liderança e tomada de decisão definido pelo grau de participação que pode variar de acordo com os níveis de cooperação e participação (Maes \& Van Hootergem, 2011).

Frequência, impacto e planejamento da mudança (Rafferty \& Griffin, 2006; Rafferty \& Restubog, 2010) são atributos que influenciam o estresse e a forma como os indivíduos lidam com a mudança. O histórico anterior ao processo de mudança, o grau de risco e as perspectivas futuras de novos processos de mudança são atributos associados ao comprometimento dos empregados durante a mudança organizacional (Cunningham, 2006) e ao bem-estar e à justiça (Kalimo, Taris, \& Schaufeli, 2003), e à abertura dos indivíduos para aceitar o processo de mudança e emitir os comportamentos desejados (Devos, Buelens, \& Bouckenooghe, 2007), além de moderar a relação entre conteúdo e processo da mudança e as crenças, atitudes e intenções dos indivíduos (Self, Armenakis, \& Schraeder, 2007). 
As primeiras experiências e informações sobre a mudança organizacional são suficientes para trazer à tona várias cognições e sentimentos como frustração, entusiasmo ou medo (Bouckenooghe, 2010; Choi, 2011; Lines, 2005). Por outro lado, as mudanças podem ser interpretadas de forma positiva quando eliminam o retrabalho, rotinas desnecessárias e maximizam as oportunidades de crescimento e desenvolvimento para os funcionários (Kruglanski, Higgins, \& Capozza, 2007).

Quando a organização planeja a mudança com antecedência, são fornecidas mais informações às pessoas sobre a iminência da mudança e sua provável duração (Nery \& Neiva, 2015; Neiva, Odelius, $\&$ Ramos, 2015). Além disso, os empregados tendem a perceber a mudança como menos ameaçadora quando há planejamento prévio para sua implementação (Cunningham, 2006; Devos et al., 2007), influenciando os sentimentos e pontos de vista das pessoas sobre a mudança (Vakola, Tsaousis, \& Nikolaou, 2004). A ocorrência de atitudes positivas - atitudes de aceitação - durante o processo de mudança facilitaria a ocorrência de comportamentos para ajustar indivíduos às mudanças, aumentando a possibilidade de iniciativas bem-sucedidas (Nery \& Neiva, 2015; Neiva \& Paz, 2012). As atitudes negativas, por exemplo, o medo e o ceticismo em relação às alterações podem reduzir a probabilidade de comportamentos de apoio dos empregados (Neiva \& Paz, 2012).

A influência do planejamento das mudanças organizacionais e o grau de risco percebido pelos empregados nas avaliações sobre o processo de mudança (Cunningham, 2006; Devos et al., 2007; Nery \& Neiva, 2015; Neiva et al., 2015) subsidiaram as hipóteses 1 e 2 :

H1. A relação entre o planejamento da mudança organizacional e a atitude de aceitação frente à mudança organizacional será positiva e a relação entre as atitudes de temor e ceticismo, negativa.

H2. A relação entre o planejamento da mudança será positiva com comportamento de apoio à mudança organizacional e negativa com resistência.

De modo complementar, a literatura indica que a percepção do risco e impacto decorrente das mudanças organizacionais influenciam as atitudes dos indivíduos (Nery \& Neiva, 2015; Nery, Neiva, \& Mendonça, 2016). Assim, é provável que indivíduos que percebem um maior risco decorrente da mudança, seja por perdas de benefícios ou vantagens no contexto organizacional, vivenciem sensações de incerteza e instabilidade apresentando atitude de temor. Considerando a percepção de risco e impacto das mudanças organizacionais, portanto estabelece-se a terceira hipótese:

H3. A percepção de risco e impacto das mudanças organizacionais relaciona-se positivamente às atitudes de temor e de ceticismo frente às mudanças e negativamente à atitude de aceitação.

Devos, et al. (2007) afirmam que a percepção do grau de risco da mudança influencia a abertura dos indivíduos para aceitar o processo de mudanças e para emitir os comportamentos desejados. Assim, se o nível percebido de risco é alto os indivíduos não se envolvem nas mudanças promovidas pela organização. Por isso, é importante identificar os riscos da mudança para reduzir o nível de risco percebido, proporcionando um ambiente favorável em que os indivíduos se sintam habilitados a assumir riscos (Fevre, 2014). Embora Ajzen e Fisbein (1980) afirmem que o afeto e a cognição podem ou não eliciar comportamentos posteriores, estudos mais recentes indicam que os comportamentos de resistência à mudança têm gênesis na crença de que os custos pessoais da mudança irão sobrepor seus benefícios (Nery et al., 2016; Vakola, 2016). Assim, hipotetizou-se:

H4. A relação entre risco das mudanças organizacionais e resistência à mudança será positiva e a relação entre risco e comportamento de apoio às mudanças será negativa.

Algumas proposições da literatura (George \& Jones, 2001; Lines, 2005; Oreg et al., 2011) indicam que indivíduos com atitudes positivas frente à mudança têm expectativas positivas para os resultados da mudança, por isso tendem a demonstrar apoio às mudanças, porque estas facilitam a adaptação às exigências da mudança organizacional (Bordia et al., 2011; Choi, 2011; Neiva \& Paz, 2012).

Valley e Thompson (1998) afirmam que a resistência, entendida como resposta comportamental, é fortalecida quando as atitudes em relação à mudança são negativas, ou a competência e segurança dos indivíduos estão sob ameaça. Sarnoff, Katz e McClintock (1965, citados por Valley \& Thompson, 1998) afirmam que atitudes moldam sentimentos das pessoas e suas percepções em relação à mudança ou ao objeto novo, e moldam o comportamento em direção à mudança ou ao objeto novo. Tomando por base a Teoria do comportamento planejado (Ajzen, 2001, 1991; Kim et al., 2011) que postula que o comportamento humano é uma função decorrente das atitudes, das normas subjetivas e da percepção sobre o controle comportamental, é possível considerar que as atitudes ajudam as pessoas a 
tomar consciência das novas faces do ambiente, ligando cada novo item a um conjunto de crenças que transmitem valor e dirigem o comportamento (Jimmieson, Peach, \& White, 2008; Kim et al., 2011).

Em momentos de mudança, se os membros da organização abraçam a mudança ou acreditam que ela traz benefícios, eles provavelmente se adaptarão rapidamente ao novo sistema de trabalho. Atitudes negativas em direção à mudança podem ter o efeito oposto, diminuir a probabilidade de que a nova estrutura social será adaptada da forma que foi planejada (Jimmieson et al., 2008; Kim et al., 2011). Tomando por base que as atitudes podem influenciar a emissão de comportamentos (Ajzen, 1991, 2001), foram formuladas as seguintes hipóteses:

H5. A atitude de aceitação à mudança relaciona-se positivamente ao comportamento de apoio e negativamente à resistência.

H6. As atitudes de temor e ceticismo são positivamente relacionadas à resistência e negativamente ao comportamento de apoio.

As atitudes dos indivíduos frente à mudança desempenham um papel fundamental na relação entre indivíduos e mudança organizacional. Muitas vezes também citadas como facilitadores de mudança, as atitudes dos indivíduos frente à mudança organizacional adquirem caráter fundamental quando se assinala seu papel no sucesso dos processos de mudança organizacional. Buscando investigar essa relação, foi elaborada a seguinte hipótese:

H7. Atitudes frente à mudança medeiam parcialmente a relação entre os atributos da mudança e as respostas comportamentais.

Um grande desafio do campo de estudos da mudança organizacional é a investigação do tempo
(Bartunek \& Woodman, 2015; Pettigrew, Woodman, $\&$ Cameron, 2001). A literatura indica que a mudança é um fenômeno complexo. Buscando investigar o efeito moderador do tempo, utilizou-se do mecanismo psicológico subjacente que são as mudanças graduais nos modelos cognitivos dos indivíduos em seu relacionamento com a empresa proposta por Kim et al. (2011), apresentada na hipótese:

H8. O tempo exerce efeito de moderação no modelo proposto de forma que haverá diferença significativa em T1, T2 e T3.

\section{Modelo de Investigação Proposto}

O presente estudo tem como variável antecedente os atributos de mudança organizacional, como variável mediadora a atitude frente à mudança e a variável critério são as respostas comportamentais à mudança organizacional. A Figura 1 apresenta o modelo de investigação do estudo de forma gráfica, considerando à esquerda as varáveis antecedentes, no meio as variáveis mediadoras e à direita às variáveis consequentes. A figura retrata as relações expressas pelas hipóteses estabelecidas na presente pesquisa, tendo os atributos (características do contexto) de mudança organizacional como variáveis exógenas. Os atributos, por sua vez, afetam as atitudes dos indivíduos em relação à mudança, além de influenciar o modo como os indivíduos percebem e sentem o processo de mudança, construindo reações avaliativas que podem afetar ou não a forma com que eles se comportam. A suposição é a de que reações afetivas e avaliativas positivas afetam os comportamentos de apoio ao processo de mudança e aquelas reações afetivas e avaliativas negativas podem favorecer a resistência à mudança.

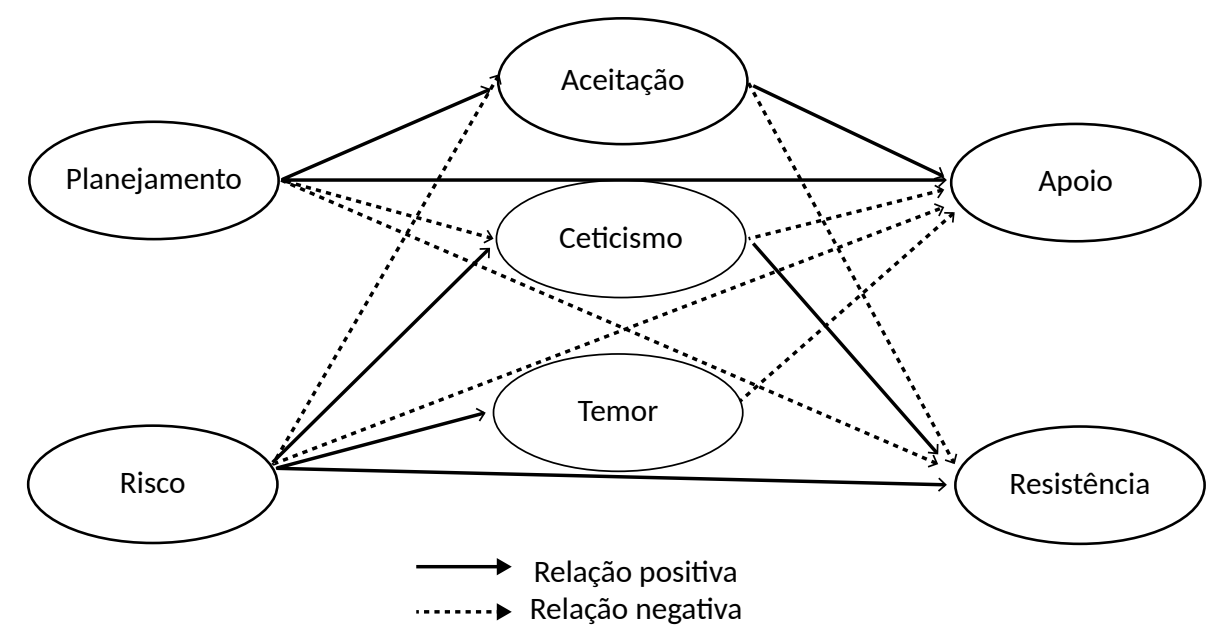

Figura 1. Modelo de Investigação do Estudo. 


\section{Método}

\section{Participantes}

A amostra da organização pública foi definida a partir da amostragem randômica estratificada baseada em 11 estados. Foi atribuído um número para cada sujeito e realizado o sorteio aleatório eletrônico utilizando - software online www.random.org - para obter amostras adequadas de cada estrato da população (Rea \& Parker, 1997). Na primeira coleta de dados foram aproveitados 544 questionários com as informações demográficas as respostas às três escalas. Na segunda fase, permaneceram na pesquisa 408 participantes. Na última etapa, permaneceram 361 respondentes. A Tabela 1 apresenta o perfil demográfico da pesquisa.

Tabela 1. Perfil dos Participantes da Pesquisa

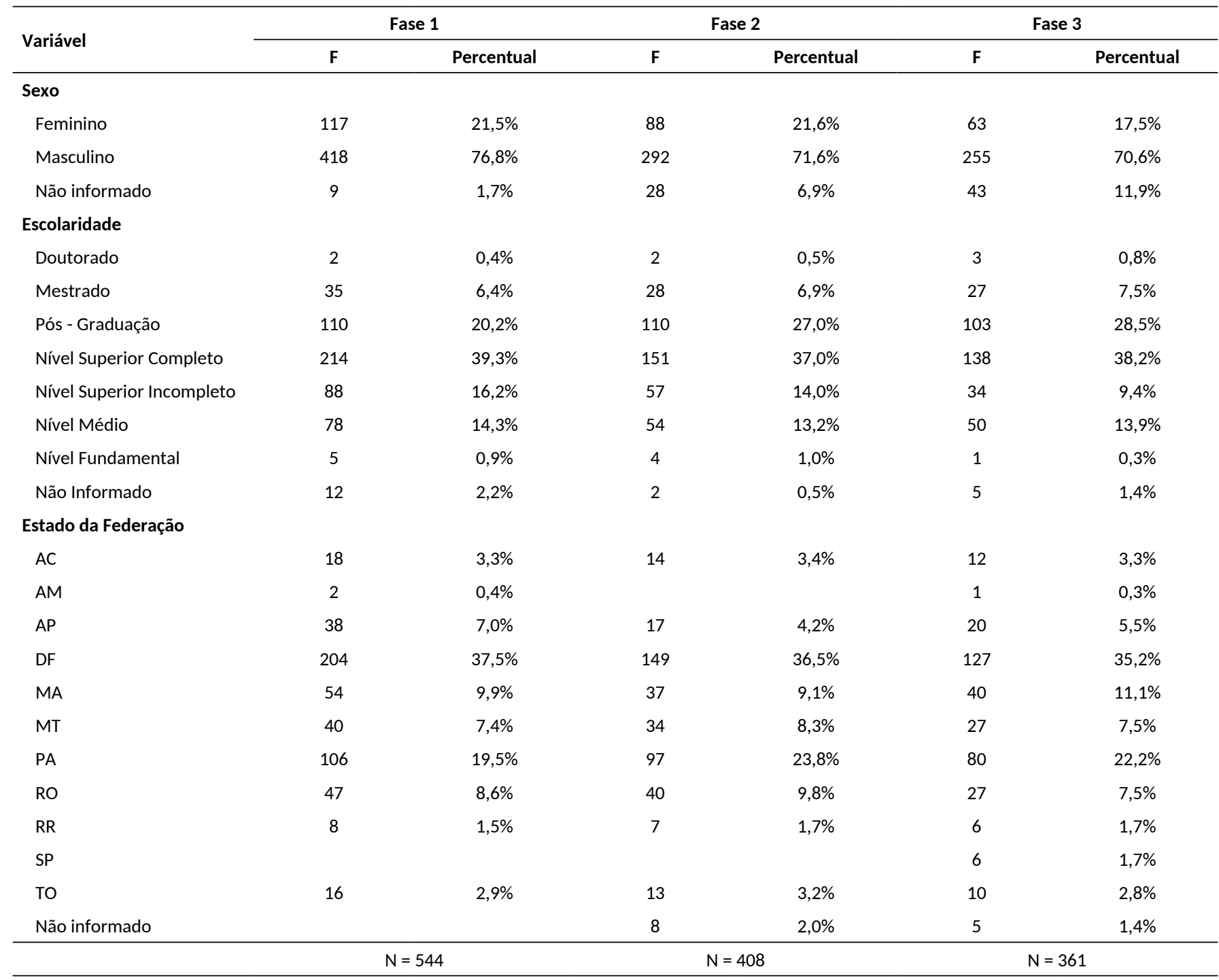

\section{Instrumentos}

Neste estudo foram aplicados três instrumentos, utilizando a escala do tipo Likert de 11 pontos 0 (discordo totalmente) a 10 (concordo totalmente). O primeiro instrumento aplicado foi a escala de atributos da mudança organizacional, elaborada por Nery e Neiva
(2015), com estrutura bifatorial. O fator planejamento, preparação e experiências anteriores à mudança PPEAM diz respeito às percepções dos empregados sobre as preparações que ocorreram antes da implantação das mudanças, como, por exemplo, o histórico e a frequência de mudanças e as experiências anteriores 
de sucesso ou fracasso. Já o fator risco da mudança relaciona-se à percepção dos empregados sobre os aspectos decorrentes da implantação da mudança que podem afetar tanto a organização quanto o próprio indivíduo. A Escala possui 21 itens com cargas fatoriais acima de 0,32 e alfas de Cronbach acima de 0,74.

O segundo instrumento é a escala de atitudes frente à mudança organizacional, validada por Neiva, Ros, \& Paz (2005), que acessa crenças, comportamentos e sentimentos dos indivíduos durante os processos de mudança organizacional, em três fatores: ceticismo, temor e aceitação. O ceticismo abrange crenças e sentimentos de descrédito relacionados ao processo de mudança. O temor é caracterizado pelo medo de perder poder, benefícios ou das incertezas vivenciadas pelos indivíduos. Atitudes de aceitação se relacionam às crenças, sentimentos e intenções comportamentais que fazem uma avaliação positiva da mudança organizacional. Este instrumento é composto por 46 itens, cargas fatoriais acima de 0,30 e Alfa de Cronbach acima de 0,85.

O terceiro instrumento, a escala de respostas comportamentais à mudança (Nery \& Neiva, 2015), possui estrutura bifatorial e 19 itens. Os instrumentos foram submetidos à análise fatorial confirmatória via equações estruturais e apresentaram bons índices de ajuste de acordo com Tabachnick e Fidell (2013).

\section{Procedimento de Coleta de Dados}

A organização disponibilizou uma listagem contendo os nomes e-mails dos sujeitos. Após a seleção dos sujeitos, os dados foram cadastrados no software online Lime Survey. A coleta de dados estruturada foi realizada em três etapas. O esquema temporal das coletas de dados está representado graficamente na Figura 2.

\begin{tabular}{|c|c|c|}
\hline $\begin{array}{l}\text { Primeira etapa } \\
\text { de coleta } \\
\text { de dados }\end{array}$ & $\begin{array}{l}\text { Segunda etapa } \\
\text { de coleta } \\
\text { de dados }\end{array}$ & $\begin{array}{c}\text { Terceira etapa } \\
\text { de coleta } \\
\text { de dados }\end{array}$ \\
\hline 2011 & $\stackrel{2012}{\uparrow}$ & 2014 \\
\hline
\end{tabular}

Figura 2. Esquema Temporal das Coletas de Dados.

Em todas as coletas de dados (T1, T2 e T3), os instrumentos de pesquisa foram encaminhados aos mesmos sujeitos, por meio de correio eletrônico, que continha um texto explicativo, o endereço online da pesquisa e a senha para acesso ao questionário.

Antes de acessar os instrumentos, os respondentes tiveram acesso ao Termo de Consentimento Livre e Esclarecido - TCLE, contendo o objetivo da pesquisa e solicitando o consentimento do participante em ser identificado. As instruções de preenchimento foram incluídas como parte dos questionários.

\section{Procedimento de Análise de Dados}

A verificação dos pressupostos estatísticos (Hair, Black, Babin, Anderson, \& Tatham, 2009; Tabachnick \& Fidell, 2013) e a verificação da estrutura fatorial dos instrumentos, de forma separada, para as amostras coletadas em T1, T2 e T3 foram os procedimentos iniciais.

Em seguida foi verificado o ajuste do modelo de investigação nos três bancos após o procedimento de limpeza de dados. Nos modelos, os construtos foram verificados por meio das médias de seus fatores. Os modelos foram estimados no programa AMOS 20 por máxima verossimilhança, para verificação do ajuste dos modelos a partir dos indicadores apontados por Byrne (2010) e Kline (2010): GFI (Goodness-of-Fit Index), CFI (Comparative Fit Index) e RMSEA. O modelo proposto foi comparado a modelos alternativos, pois tal prática constitui teste mais rigoroso do que a análise isolada do ajuste (Hair et al., 2009).

O modelo de mediação deste estudo é complexo por ter mais de uma variável como mediadora (Preacher, Zyphur, \& Zhang, 2010). Assim, verificação das hipóteses de mediação foi feita por meio de equações estruturais, verificadas a magnitude e significância dos efeitos indiretos por meio de bootstrapping. Os efeitos indiretos foram estimados a partir de uma amostra da população, produtos das estimativas dos coeficientes de regressão. Efeitos indiretos significativos são uma medida análoga à utilização de outros métodos que indicam a significâncias dos efeitos de interação da regressão (Preacher \& Selig, 2012). Para isso, foram realizadas várias análises com submodelos com o objetivo de verificar o sinal, magnitude e significância dos coeficientes, além de verificar a relação de mediação (Preacher \& Selig, 2012; Preacher et al., 2010).

Por fim, foi verificado o efeito moderador do tempo pela comparação do ajuste do modelo de investigação em T1, T2 e T3, utilizando a estatística qui-quadrado. A mudança não significativa no valor do qui-quadrado, ou $\Delta \mathrm{x}^{2}(\Delta d f$, indica que o modelo não varia ao longo do tempo, ou seja, não há efeito da moderação. Caso ocorra uma mudança significativa há evidência do efeito moderador de tempo. Também foram realizados testes $t$ de estudantes para fornecer tamanhos de efeitos para a extensão da moderação ( $d$ de Cohen). 


\section{Resultados}

Os resultados são apresentados inicialmente considerando o ajuste do modelo e na sequência são apresentados os resultados relacionados às hipóteses testadas.

\section{Modelo Estrutural e Teste de Hipóteses}

A verificação do ajuste do modelo foi realizada de forma separada para os grupos (T1, T2 e T3). Os índices de ajuste do modelo estrutural proposto em T1, T2 e T3 são considerados razoáveis e são apresentados na Tabela 2.

Tabela 2. Índices de Adequação do Modelo

\begin{tabular}{|c|c|c|c|c|c|c|c|c|c|c|c|}
\hline \multicolumn{12}{|c|}{ Ajuste do Modelo } \\
\hline & $x^{\prime \prime}$ & g.l. & $\chi^{\prime \prime} \gamma . \lambda$. & NFI & TLI & CFI & GFI & AGFI & $\begin{array}{l}\text { RMSEA } \\
\text { (IC 95\%) }\end{array}$ & $\Delta \mathrm{x}^{2 *}$ & $\Delta g . I$. \\
\hline Modelo $\mathrm{T} 1$ reespecificado & 14,77 & 3 & 4,92 & 0,99 & 0,95 & 0,99 & 0,99 & 0,92 & $\begin{array}{c}0,08 \\
(0,07-0,09)\end{array}$ & 231,41 & 2 \\
\hline Modelo $\mathrm{T} 2$ reespecificado & 12,63 & 3 & 4,21 & 0,99 & 0,94 & 0,99 & 0,99 & 0,91 & $\begin{array}{c}0,89 \\
(0,07-0,09)\end{array}$ & 138,11 & 2 \\
\hline Modelo Inicial T3 & 184,06 & 5 & 36,81 & 0,84 & 0,35 & 0,84 & 0,88 & 0,36 & $\begin{array}{c}0,31 \\
(0,28-0,34)\end{array}$ & & \\
\hline Modelo $\mathrm{T} 3$ reespecificado & 9,20 & 2 & 4,60 & 0,99 & 0,93 & 0,99 & 0,99 & 0,90 & $\begin{array}{c}0,10 \\
(0,08-0,109)\end{array}$ & 174,86 & 3 \\
\hline
\end{tabular}

Nota. ${ }^{*} p<0,001 ;$ g.I. = graus de liberdade; IC = intervalo de confiança (95\%); $\Delta \chi^{2}=$ diferença de qui-quadrado; $\Delta g . I .=$ diferença de graus de liberdade.

A primeira hipótese foi parcialmente corroborada. O efeito da relação entre planejamento da mudança organizacional e a atitude de aceitação frente à mudança organizacional é alto, positivo e significativo T1 ( $\beta=0,73, p<0,001)$, T2 $(\beta=0,72, p<0,001)$ e T3 $(\beta=0,69, p<0,001)$. O efeito da relação entre planejamento da mudança organizacional e a atitude de ceticismo é negativo T1 $(\beta=-0,36, p<0,001)$, T2 $(\beta=0,31, p<0,001)$ e T3 $(\beta=0,31, p<0,001)$. O efeito da relação entre planejamento e as atitudes de temor é baixo e significativo em $(\beta=0,13, p<0,001)$, T2 ( $\beta=0,16, p<0,001)$, mas deixa de ser negativo e significativo em T3 ( $\beta=0,03, n s)$. A segunda hipótese foi totalmente corroborada, pois o efeito do planejamento da mudança sobre os comportamentos de apoio foram positivos, altos e significativos nos três momentos T1 $(\beta=0,60, p<0,001)$, T2 $(\beta=0,54, p<0,001)$ e T3 $(\beta=0,55, p<0,001)$. Contudo, o relacionamento entre planejamento e resistência teve efeito negativo fraco em T1 $(\beta=-0,24, p<0,001)$ e T2 $(\beta=-0,12, p<0,05)$ e não significativo em T3 ( $\beta=-0,07, n s)$.

A terceira hipótese foi parcialmente corroborada. A variável risco da mudança organizacional relaciona-se positivamente com efeito médio e significativo às atitudes de temor em T1 $(\beta=0,49, p<0,001)$, T2 ( $\beta=0,45$, $p<0,001)$ e T3 $(\beta=0,50, p<0,001)$. O efeito da relação entre risco e ceticismo é médio e positivo em T1 $(\beta=0,35$, $p<0,001)$, T2 $(\beta=0,33, p<0,001)$ e T3 $(\beta=0,37$, $p<0,001)$. Entretanto o efeito da relação entre as variáveis risco da mudança e atitude de aceitação é baixo, em T1 ( $\beta=-0,001, n s)$ e T3 ( $\beta=-0,092, n s)$ e significativo somente em T2 $(\beta=-0,105, p<0,05)$.

A quarta hipótese foi parcialmente corroborada. Em T1 o efeito do risco sobre a variável apoio é baixo, negativo e significativo T1 $(\beta=-0,11, p<0,05)$. Contudo, o efeito decresce de valor e passa a ser não significativo em T2 ( $\beta=-0,06, n s)$ e T3 ( $\beta=-0,08, n s)$. $O$ efeito da relação risco e resistência é positivo e significativo em T1 ( $\beta=0,31, p<0,05)$, T2 $(\beta=0,21, p<0,001)$ e T3 $(\beta=0,23, p<0,001)$.

A quinta hipótese previa que a atitude de aceitação à mudança teria relacionamento positivo com comportamento de apoio e negativo com resistência. De fato, a relação entre atitude de aceitação à mudança foi positiva e significativa em T1 ( $\beta=0,23, p<0,001)$, T2 $(\beta=0,57, p<0,001)$ e T3 $(\beta=0,64, p<0,001)$. Contudo, a relação entre a atitude de aceitação e resistência foi positiva em T1 $(\beta=0,12, p<0,05)$ e não foi significativa em T2 $(\beta=-0,09, n s)$ e T3 $(\beta=-0,20, n s)$.

A sexta hipótese foi corroborada. A relação entre temor e resistência é positiva e significativa em T1 $(\beta=0,45, p<0,001)$, T2 $(\beta=0,30, p<0,001)$ 
e T3 $(\beta=0,47, p<0,001)$. O efeito da relação entre ceticismo e resistência é alto, positivo, e significativo em $\mathrm{T} 1(\beta=0,70, p<0,001), \mathrm{T} 2(\beta=0,72, p<0,05)$ e T3 $(\beta=0,72$, $p<0,001)$. O efeito da relação entre as atitudes de temor e comportamento de apoio são baixas e negativas em T1 $(\beta=-0,17, p<0,001)$, T2 $(\beta=-0,11, p<0,001)$ e T3 $(\beta=-0,19, p<0,001)$. O efeito da relação entre as atitudes de ceticismo e o comportamento de apoio também são negativas e significativas T1 $(\beta=-0,24, p<0,001)$, T2 $(\beta=-0,16, p<0,001)$ e T3 $(\beta=-0,16, p<0,001)$.

A sétima hipótese se relacionava ao teste de mediação. A literatura afirma que quando a variável mediadora é incluída na equação de regressão, há redução ou é neutralizado o impacto da variável independente sobre a dependente (Tabachnick \& Fidell, 2001). Os resultados das análises de mediação são apresentados nas Tabelas 3 e 4.

Tabela 3. Resultado do Teste de Mediação para a Variável Dependente Comportamento de Apoio às Mudanças Organizacionais

\begin{tabular}{|c|c|c|c|c|c|c|}
\hline \multirow[b]{3}{*}{ Variáveis } & \multicolumn{6}{|c|}{ Apoio } \\
\hline & \multicolumn{2}{|c|}{$\mathrm{T} 1$} & \multicolumn{2}{|c|}{$\mathrm{T} 2$} & \multicolumn{2}{|c|}{ T3 } \\
\hline & $\beta$ & $\begin{array}{c}\text { Efeito } \\
\text { indireto }\end{array}$ & $\beta$ & $\begin{array}{c}\text { Efeito } \\
\text { indireto }\end{array}$ & B & $\begin{array}{c}\text { Efeito } \\
\text { indireto }\end{array}$ \\
\hline Planej. & $0,60^{*}$ & & $0,54^{*}$ & & $0,55^{*}$ & \\
\hline Risco & $-0,11^{* *}$ & & $-0,06 \mathrm{~ns}$ & & $-0,08 \mathrm{~ns}$ & \\
\hline \multicolumn{7}{|l|}{ Planej. $\rightarrow$} \\
\hline Aceitação & $0,43^{*}$ & $0,25^{* *}$ & $0,11^{* *}$ & $0,53^{* *}$ & $0,10^{* *}$ & $0,52^{* *}$ \\
\hline \multicolumn{7}{|l|}{ Planej. $\rightarrow$} \\
\hline Temor & $0,58^{*}$ & $0,02^{* *}$ & $0,52^{*}$ & $0,01^{* *}$ & $0,56^{*}$ & $-0,006 n s$ \\
\hline \multicolumn{7}{|l|}{ Planej. $\rightarrow$} \\
\hline Ceticismo & $0,51^{*}$ & $0,08^{* *}$ & $0,49^{*}$ & $0,05^{* *}$ & $0,51^{*}$ & $0,04^{* *}$ \\
\hline \multicolumn{7}{|l|}{ Risco $\rightarrow$} \\
\hline Aceitação & $-0,11^{*}$ & $0,00 \mathrm{~ns}$ & $0,006 \mathrm{~ns}$ & $-0,06 \mathrm{~ns}$ & $-0,01 n s$ & $-0,06 \mathrm{~ns}$ \\
\hline \multicolumn{7}{|l|}{ Risco $\rightarrow$} \\
\hline Temor & $0,015 \mathrm{~ns}$ & $-0,12^{* *}$ & 0,03 ns & $-0,09^{* *}$ & $0,015 \mathrm{~ns}$ & $-0,09^{* *}$ \\
\hline \multicolumn{7}{|l|}{ Risco $\rightarrow$} \\
\hline Ceticismo & $0,04 \mathrm{~ns}$ & $-0,16^{* *}$ & $0,04 n s$ & $-0,11^{* *}$ & 0,05 ns & $-0,13^{* *}$ \\
\hline
\end{tabular}

Os resultados do teste de mediação das atitudes frente à mudança organizacional para a variável comportamento de apoio à mudança organizacional demonstram que a aceitação de mudança é a única variável que medeia (parcialmente) a relação entre planejamento e comportamentos de apoio em T1, T2 e T3. As demais relações de mediação não foram constantes ao longo do tempo. A relação entre planejamento e apoio à mudança teve mediação parcial em T1 e T2, mas não em T3. Embora, os indicadores de T3 dessa relação demonstrassem indícios de mediação (Baron \& Kenny, 1986), os efeitos indiretos indicam que não há relação de mediação.

Os indicadores das relações entre risco e comportamento de apoio demonstraram mediação completa pelas variáveis temor e ceticismo em $\mathrm{T} 1$, ou seja, a relação entre a variável preditora e a variável critério se tornou insignificante após a inclusão da variável mediadora (Hair et al, 2010). Contudo, essas mesmas relações não tiveram índices que indicavam mediação em T2 e T3, de acordo com os critérios de Baron e Kenny (1986). Contudo, os efeitos indiretos apresentaram magnitude baixa, mas significativa. Considera-se que isso seja um viés da presente amostra, que foi evidenciada pelo uso da técnica de bootstrapping. Ou seja, é possível que nessa amostra realmente não ocorra mediação, mas, que essa relação de mediação pode ocorrer em outras amostras, conforme ficou evidenciado nas relações de dessas variáveis, em T1.

Tabela 4. Resultado do Teste de Mediação para a Variável Dependente Resistência à Mudança

\begin{tabular}{|c|c|c|c|c|c|c|}
\hline \multirow[b]{3}{*}{ Relação } & \multicolumn{6}{|c|}{ Resistência } \\
\hline & \multicolumn{2}{|c|}{$\mathrm{T} 1$} & \multicolumn{2}{|c|}{$\mathrm{T} 2$} & \multicolumn{2}{|c|}{ T3 } \\
\hline & $\beta$ & $\begin{array}{c}\text { Efeito } \\
\text { indireto }\end{array}$ & $\beta$ & $\begin{array}{c}\text { Efeito } \\
\text { indireto }\end{array}$ & B & $\begin{array}{l}\text { Efeito } \\
\text { indiretc }\end{array}$ \\
\hline Planej. & $-0,24^{*}$ & & $-0,12^{* *}$ & & $-0,07 \mathrm{~ns}$ & \\
\hline Risco & $0,31^{*}$ & & $0,21^{*}$ & & $0,23^{*}$ & \\
\hline \multicolumn{7}{|l|}{ Planej. $\rightarrow$} \\
\hline Aceitação & $-0,34^{*}$ & $0,17 n s$ & $-0,05 \mathrm{~ns}$ & $0,02 n s$ & 0,06 ns & $-0,03^{* *}$ \\
\hline \multicolumn{7}{|l|}{ Planej. $\rightarrow$} \\
\hline Temor & $-0,18^{*}$ & $-0,06^{* *}$ & $-0,06 \mathrm{~ns}$ & $-0,05^{* *}$ & $-0,09$ ns & 0,01 ns \\
\hline \multicolumn{7}{|l|}{ Planej. $\rightarrow$} \\
\hline Ceticismo & $0,01 \mathrm{~ns}$ & $-0,26^{* *}$ & $0,10^{* *}$ & $-0,22^{* *}$ & $0,05 \mathrm{~ns}$ & $-0,13^{* *}$ \\
\hline \multicolumn{7}{|l|}{ Risco $\rightarrow$} \\
\hline Aceitação & $0,31^{*}$ & $0,00 \mathrm{~ns}$ & $0,19^{*}$ & $0,01^{* *}$ & $0,22^{*}$ & $0,01 \mathrm{~ns}$ \\
\hline \multicolumn{7}{|l|}{ Risco $\rightarrow$} \\
\hline Temor & $0,09^{* *}$ & $0,22^{* *}$ & $0,07 n s$ & $0,13^{* *}$ & $0,00 \mathrm{~ns}$ & $0,23^{* *}$ \\
\hline \multicolumn{7}{|l|}{ Risco $\rightarrow$} \\
\hline Ceticismo & $0,07 \mathrm{~ns}$ & $0,23^{* *}$ & $-0,02$ ns & $0,23^{* *}$ & $-0,03 n s$ & $0,27^{* *}$ \\
\hline
\end{tabular}

Os resultados do teste de mediação das atitudes frente à mudança organizacional para a variável resistência à mudança organizacional demonstram que a aceitação de mudança não medeia a relação entre planejamento e resistência em T1, T2 e T3. Contudo, embora em T3, os índices de efeitos indiretos demonstrem ocorrer mediação, a relação não foi encontrada na presente amostra. 
As atitudes de temor e ceticismo medeiam a relação entre planejamento e resistência em T1 e T2 de forma significativa, mas não em T3. A relação entre variável risco e resistência, com a inclusão da variável aceitação no modelo de mediação é significativa em T2 ( $\beta=0,19$, $p<0,001$ ) indicando mediação parcial, mas em T1 e T3 não há indícios relação de mediação.

A atitude de temor medeia parcialmente a relação entre risco da mudança e resistência em T1 $(\beta=0,09$, $p<0,001)$, e essa relação de mediação passa a ser completa em T2 $(\beta=0,07, p<0,001)$ e T3 $(\beta=0,00$, $p<0,001)$. A atitude de ceticismo frente à mudança organizacional apresenta índices que confirmam a relação de mediação completa em T1, T2 e T3.

A partir dos dados apresentados nas Tabelas $14 \mathrm{e}$ 15 é possível afirmar que a oitava hipótese que postulava que existia relação de mediação parcial das atitudes frente à mudança na relação entre os atributos da mudança e as respostas comportamentais. Contudo, de acordo com as análises de dados essa relação foi parcialmente corroborada.

Para o teste da última hipótese desse estudo foi feita análise do efeito de moderação do tempo. Hair et al. (2010) afirmam que o efeito moderador decorre da inclusão de uma terceira variável ou construto que modifica a relação entre duas variáveis relacionadas. A nona hipótese prediz que o tempo exerce efeito de moderação de tal forma que os índices de ajuste do modelo diminuem ao longo do tempo. Para comparar o modelo nos três grupos (T1, T2 e T3) foi utilizado o índice de alteração do qui-quadrado, ou $\Delta \mathrm{x}^{2}(\Delta d f)$. O valor de alteração não foi significativo entre T1 e T2, nem entre T2 e T3 indicando que não houve efeito moderador do tempo no modelo. Contudo, houve diferença significativa entre T1 e T3 de um grau de liberdade e a alteração do qui-quadrado foi marginalmente significativa $\Delta x^{2}(1)=3,43, p=0,06$ diferença entre, assim é possível afirmar que a hipótese 9 não foi corroborada. Em suma, o tempo não modera as relações apresentadas pela pesquisa, o que indica que as relações postuladas permanecem estáveis durante os três momentos de coleta de dados.

\section{Discussão e conclusões}

A grande contribuição do estudo diz respeito à identificação dos efeitos das características/atributos do processo de mudança sobre as atitudes positivas e negativas em relação às mudanças e sobre os comportamentos de apoio e resistência a mudanças. O modelo de investigação obteve bons índices de ajuste em T1, T2 e T3, o que indica que a resposta comportamental de apoio à mudança depende da percepção de planejamento das mudanças organizacionais e que a resistência depende da percepção de risco da mudança. Esse dado reforça, por meio de um modelo quantitativo, a literatura que indicava a influência dos atributos da mudança organizacional nas respostas comportamentais (By, 2005; Kalimo et al., 2003; Kim et. al, 2011; Maes \& Hootergem, 2011; Oreg et al., 2011; Vakola, 2016; Vakola et al., 2013) . Esse dado também dá suporte ao processo de planejamento, preparo e concepção das ações das intervenções como fatores primordiais nas respostas positivas dos empregados.

Os atributos planejamento e risco da mudança também demonstraram influência nas atitudes frente à mudança organizacional, tal como proposto por Self et al. (2007) e verificado nos estudos de Nery e Neiva (2015) e Nery et al. (2016). Esse achado é relevante porque evidencia a relação entre cognições e atitudes em um contexto de mudança, com recorte longitudinal, num delineamento pouco estudado no Brasil e mundo (By, 2005; Kalimo et al., 2003; Kim et al., 2011; Maes \& Hootergem, 2011; Oreg et al., 2011; Vakola, 2016; Vakola et al., 2013). As análises apontaram que o atributo risco da mudança teve efeito baixo, mas significativo em resistência à mudança. Isso significa que quanto maior o risco percebido da mudança, maior será a resistência dos indivíduos. Esse achado corrobora a literatura indicando que o grau de risco das mudanças tem relação com a abertura dos indivíduos para aceitar o processo de mudanças e emitir os comportamentos desejados (Devos et al., 2007). É importante ressaltar que a maioria dos efeitos são consistentes nos três momentos, com exceção dos efeitos do atributo de risco da mudança sobre os comportamentos de apoio que apresentam gradação/diminuição ao longo do tempo, podendo estar relacionada ao fato de a organização não ter investido em ações de planejamento e preparo e acompanhamento dos recursos humanos durante o processo de mudança. Os resultados do presente estudo fornecem suporte consistente para a importância das crenças e emoções positivas (atitudes) sobre a mudança como antecedentes do comportamento positivo e negativo frente à mudança. Esse resultado corrobora diversos autores do tema (Bordia et al., 2011; Fugate et al., 2012; Oreg et al., 2011). Foi apresentada a influência das atitudes frente à mudança organizacional (cognições avaliativas e afetos) nos comportamentos dos empregados que tornam possível o sucesso ou fracasso dos programas e mudanças (Choi, 2011). Os resultados permitem verificar que de fato há influência 
das atitudes negativas à mudança - temor e ceticismo na ocorrência de comportamentos de resistência às mudanças (Lines, 2005; Nery \& Neiva, 2015; Neiva \& Paz, 2012; Oreg et al., 2011). As atitudes investigadas estão de acordo com os modelos psicológicos sociais que predizem a consistência de comportamento-atitude (Fazio, 1986; Glasman \& Albarracin, 2006). Pesquisas anteriores sobre comprometimento com a mudança (Herscovitch \& Meyer, 2002) sugeriram que atitudes de mudança podem ser preditores mais fortes de comportamentos de apoio mais discricionários à mudança. Outro fator importante está relacionado ao papel mediador das atitudes de aceitação frente ao planejamento da mudança e aos comportamentos de apoio a mudança.

Apesar de os resultados apontarem que as crenças e afetos predizem comportamentos específicos, os resultados das atitudes de aceitação sobre o comportamento de resistência foram inconclusivos, na medida em que foi fraca, mas positiva em T1 $(\beta=0,12, p<0,05)$ e não foi significativa em T2 ( $\beta=-0,09, n s)$ e T3 $(\beta=-0,20, n s)$. Nesse caso, pode ter havido algum problema de mensuração nos instrumentos ou a consistência de comportamento-atitude não ocorre em todas as situações. Em situações nas quais outras variáveis de contexto estejam atuando (Ajzen, 1991, 2001), por exemplo, falta de percepção de benefícios para os indivíduos no processo de mudança ou negligência por parte da alta gestão pode haver manifestação de resistência mesmo que os indivíduos apresentem crenças e afetos positivos em relação ao processo.

O teste do modelo de mediação indica que as atitudes de aceitação medeiam a relação entre planejamento e respostas comportamentais à mudança. Esse resultado vai ao encontro da literatura especializada e reforça os achados de estudos anteriores (Bordia et al., 2011; Nery \& Neiva, 2015), demonstrando a influência da atitude frente à mudança na resposta comportamental de apoio à mudança dos indivíduos. Embora esse estudo tenha identificado que as atitudes frente à mudança medeiam parcialmente a relação entre atributos e comportamentos da mudança, esse achado é relevante porque corrobora a literatura indicando a influência das atitudes frente à mudança em facilitar o sucesso dos processos de mudança organizacional (Neiva \& Paz, 2012). Para a prática do Comportamento Organizacional, esse achado é bastante relevante, considerando que mudança organizacional é um fenômeno complexo e multifacetado, e grande parte da publicação se caracteriza pelos poucos dados empíricos e pela abundância das proposições prescritivas (Choi, 2011). De acordo com os achados do estudo, as atitudes atuam diretamente, mas também atuam de maneira indireta, na medida em que são fomentadas pelo planejamento. Esse resultado configura-se como uma contribuição muito significativa, o que dá mais suporte às ações organizacionais direcionadas ao planejamento de ações e ao preparo dos indivíduos. De maneira geral, é possível inferir que ações que visem tornar a avaliação sobre o processo de mudança mais positiva são efetivos para o alcance do sucesso dos programas de mudança (Choi, 2011; Lines, 2005).

Os resultados refutaram o teste do efeito de moderação do tempo, embora Kim et al. (2011) tenham encontrado evidências do efeito moderador do tempo em seu estudo. É possível que a ausência de efeito decorra do intervalo temporal de coleta de dados que pode não ter sido adequado para identificar o efeito em uma organização pública. Assim, sugere-se que novos estudos sejam realizados buscando investigar o efeito do tempo em organizações da iniciativa privada ou de outros setores produtivos. Outra explicação pode estar relacionada a quais fatores podem contribuir para que haja alteração nessa relação. As ações realizadas pela organização durante o período de coleta de dados não estiveram relacionadas à gestão da mudança do ponto de vista humano. As ações se restringiram à preparação de nova marca e maior atuação da holding frente à empresa controlada. É importante considerar a natureza pública da organização, o que parece dificultar a concretização das mudanças (Neiva \& Paz, 2012).

Esta pesquisa é relevante porque investiga variáveis negligenciadas - atributos de mudança (planejamento, risco e tempo) - e por buscar ampliar a compreensão dos fatores influenciam a adoção das respostas comportamentais - resistência e comportamento de apoio à mudança organizacional (Shoss, Witt, \& Vera, 2011). A contribuição prática deste estudo é subsidiar gestores de mudança organizacional sobre a relevância dos atributos - planejamento, preparação e experiências anteriores à mudança e o risco da mudança - que devem ser monitorados para potencializar o sucesso da mudança organizacional.

Do ponto de vista metodológico, este estudo realizou três coletas de dados, com os mesmos sujeitos em um contexto de mudança organizacional. Considera-se que o presente trabalho atingiu seus objetivos principais e apontou importantes implicações práticas, teóricas e metodológicas, especialmente para uma maior estruturação do campo de estudos por meio da identificação e compreensão dos aspectos da mudança que influenciam as atitudes e os comportamentos dos indivíduos tal como 
preconizado por Oreg, et al. (2011), Rafferty e Griffin (2006) e Rafferty e Restubog (2010).

Como limitações da pesquisa, pode-se citar o uso de uma única fonte de coleta de dados que pode gerar a variância do método comum (Podsakoff, MacKenzie, Lee, \& Podsakoff, 2003). Os testes realizados tendo o fator único como explicador dos resultados não apresentaram índices de ajuste adequados, o que retira a hipótese de que a variância comum explique os resultados (Podsakoff et al., 2003). Para refutar de maneira contundente tal efeito, sugere-se que estudos com medidas comportamentais observacionais sejam usadas. Outra limitação está relacionada ao fato da amostra ser restrita a uma organização pública. Tal fato limita a variabilidade dos resultados e impede estudos envolvendo vários níveis de análise (ex. individual e organizacional).

Como agenda de pesquisa sugere-se que o modelo desta pesquisa seja replicado em organizações privadas para verificar se em um contexto mais competitivo há o efeito moderador do tempo e para verificar a generalização desses resultados. Sugere-se ainda investigar a relação das atitudes frente às mudanças com absenteísmo, além da adoção de triangulação de método, e realização de mais estudos que investiguem a mudança em delineamentos longitudinais (Bartunek \& Woodman, 2015).

\section{Referências}

Ajzen, I. (1991). The theory of planned behavior. Organizational Behavior and Human Decision Processes, 50(2), 179-211. doi:10.1016/0749-5978(91)90020-T

Ajzen, I. (2001). Nature and operation of attitudes. Annual Review of Psychology, 52, 27-58. doi: 10.1146/annurev.psych.52.1.27

Ajzen, I., \& Fishbein, M. (1980). Understanding attitudes and predicting social behavior. Englewood Cliffs, NJ: Prentice-Hall.

Barends, E., Janssen, B., Have, W. ten, \& Have, S. ten. (2014). Effects of change interventions: What kind of evidence do we really have? The Journal of Applied Behavioral Science, 50(1), 5-27. doi: $10.1177 / 0021886312473152$

Baron, R. M., \& Kenny, D. A. (1986). The moderator-mediator variable distinction in social psychological research: Conceptual, strategic, and statistical considerations. Journal of Personality and Social Psychology, 51(6), 1173-1182. doi:10.1037/0022-3514.51.6.1173

Bartunek, J. M., \& Woodman, R. W. (2015). Beyond Lewin: Toward a temporal approximation of organization development and change. Annual Review Organizational Psychology and Organizational Behavior, 2, 157-182. doi: 10.1146/annurev-orgpsych-032414-111353

Bordia, P., Restubog, S. L. D., Jimmieson, N., \& Irmer, B. (2011). Haunted by the past: Effects of poor change management history on employee attitudes and turnover. Group \& Organization Management, 36(2) 191-222. doi: $10.1177 \% 2 F 1059601110392990$

Bouckenooghe, D. (2010). Positioning change recipients' attitudes toward change in the organizational change literature.
The Journal of Applied Behavioral Science, 46(4), 500-531. doi: $10.1177 \% 2 F 0021886310367944$

Bryson, A., Barth E., \& Dale-Olsen, H. (2013). The effects of organizational change on worker well-being and the moderating role of trade unions. Industrial and Labor Relations Review, 66(4), 989-1011. doi: $10.1177 \% 2 F 001979391306600410$

By, R. T. (2005). Organisational change management: A critical review. Journal of Change Management. 5(4), 369- 380. doi: 10.1080/14697010500359250

Byrne, B. M. (2010). Structural equation modeling with Amos: Basic concepts, applications, and programming ( $2^{\mathrm{a}}$ ed.). Nova lorque, NY: Taylor and Francis Group.

Choi, M. (2011). Employees' attitudes toward organizational change: A literature review. Human Resource Management, 50(4), 479-500. doi: $10.1002 / \mathrm{hrm} .20434$

Cunningham, G. B. (2006). The relationships among commitment to change, coping with change, and turnover intentions. European Journal of work and Organizational Psychology, 15(1), 29-45. doi: 10.1080/13594320500418766

Dahl, M. S. (2011). Organizational change and employee stress. Management Science, 57(2), 240-256. doi: 10.1287/mnsc.1100.1273

Devos, G., Buelens, M., \& Bouckenooghe, D. (2007). The contribution of content, context, and process in understanding openness to organizational change: Two experimental simulation studies. Journal of Social Psychology, 147(6), 607-630. doi: 10.3200/SOCP.147.6.607-630

Fazio, R. H. (1986). How do attitudes guide behavior? In R. M. Sorrentino \& E. T. Higgins (Orgs.), Handbook of motivation and cognition: Foundations of social behavior (pp. 204-243). Nova lorque, NY: Guildford Press.

Fevre, D. M. (2014). Barriers to implementing pedagogical change: The role of teachers' perceptions of risk. Teaching and Teacher Education, 38, 56-64. doi: 10.1016/j.tate.2013.11.007

Fugate.M., Prussia G., \& Kinicki, A.J.(2012). Managing employee withdrawal during organizational hange: The role of threat appraisal. Journal of Management, 38(3), 890-914. doi: 10.1177\%2F0149206309352881

George, J. M., \& Jones, G. R. (2001). Towards a process model of individual change in organization. Human Relations, 54(4), 419-444. doi: $10.1177 \% 2 F 0018726701544002$

Glasman, L. R., \& Albarracin, D. (2006). Forming attitudes that predict future behavior: A meta-analysis of the attitudebehavior relation. Psychological Bulletin, 132(5), 778-822. doi: 10.1037/0033-2909.132.5.778

Hair, J. F., Black, B., Babin, B., Anderson, R. E., \& Tatham, R. L. (2009) Análise multivariada de dados (6ª ed.). Porto Alegre: Bookman.

Herscovitch, L., \& Meyer, J. P. (2002). Commitment to organizational change: Extension of a three-component model. Journal of Applied Psychology, 87(3), 474-487. doi: 10.1037/0021-9010.87.3.474

Jimmieson, N. L., Peach, M., \& White, K. M. (2008) Utilizing the Theory of Planned Behavior to inform change management: An investigation of employee intentions to support organizational change. Journal of Applied Behavioral Science, 44(2), 237-262. doi: $10.1177 / 0021886307312773$

Kalimo, R., Taris, T. W., \& Schaufeli, W. B. (2003). The effects of past and anticipated future downsizing on survivor wellbeing: An equity perspective. Journal of Occupational Health Psychology, 8(2), 91-109. doi: 10.1037/1076-8998.8.2.91 
Efeitos dos atributos do processo de mudança nas atitudes e nos comportamentos dos trabalhadores

Kim, T. G., Hornung, S., \& Rousseau, D. M. (2011) Changesupportive employee behavior: antecedents and the moderating role of time. Journal of Management, 37, 1664-1693. doi: 10.1177/0149206310364243

Kline, R. B. (2010). Principles and practice of structural equation modeling. Nova lorque: Guilford Press.

Kruglanski, A. P., A; Higgins, E; \& Capozza, D. (2007). "On the move" or "staying put": Locomotion, need for closure, and reactions to organizational change. Journal of Applied Social Psychology, 37(6), 1305-1340. doi: 10.1111/j.1559-1816.2007.00214.x

Lines, R. (2005). The structure and function of attitudes toward organizational change. Human Resource Development Review, 4, 8-32. doi: $10.1177 / 1534484304273818$

Maes, G., \& Van Hootegem, G. (2011). Toward a dynamic description of the attributes of organizational change. Research in Organizational Change and Development, 19, 191-231. doi: 10.1108/S0897-3016(2011)0000019009

Neiva, E. R., Odelius, C. C., \& Ramos, L. D. (2015). The organizational change process: Its influence on competences learned on the job. BAR, Brazilian Administration Review, 12(4), 324-347. doi: 10.1590/1807-7692bar2015140082

Neiva, E. R., \& Paz, M. G. T. (2012). Percepção de mudança individual e organizacional: o papel das atitudes, dos valores, do poder e da capacidade organizacional. Revista de Administração, 47, 22-37. doi: 10.5700/rausp1023

Neiva, E. R., Ros, M., \& Paz, M. G. T. (2005). Attitudes towards organizational change: Validation of a scale. Psychology in Spain, 9(1), 81-90. Recuperado de http://www.psychologyinspain.com/ content/full/2005/frame.asp?id=9010

Nery, V. F., \& Neiva, E. R. (2015). Variáveis de contexto e respostas à mudança organizacional: testando o papel mediador das atitudes. Psicologia: Teoria e Pesquisa, 31(2), 259-268. doi: 10.1590/0102-37722015021968259268

Nery, V. F., Neiva, E. R., \& Mendonça, H. (2016). The changing context and the organizational justice impact on the employee well-being. Paidéia, 26(65), 317-324. doi: 10.1590/1982-43272665201609

Oreg, S., Vakola, M., \& Armenakis, A. (2011). Change recipients' reactions to organizational change: A 60-year review of quantitative studies. The Journal of Applied Behavioral Science, 47(4), 461-524. doi: $10.1177 / 0021886310396550$

Pettigrew, A. M., Woodman, R. W., \& Cameron, K. S. (2001). Studying organizational change and development: challenges for future research. Academy of Management Journal, 44(4), 697-713. doi: $10.2307 / 3069411$

Podsakoff, P. M., MacKenzie, S. B., Lee, J.-Y., \& Podsakoff, N. P. (2003). Common method biases in behavioral research: A critical review of the literature and recommended remedies. Journal of Applied Psychology, 88(5), 879-903. doi: 10.1037/0021-9010.88.5.879

Preacher, K. J., \& Selig, J.P. (2012). Advantages of Monte Carlo confidence intervals for indirect effects. Communication Methods and Measures, 6, 77-98. doi: 10.1080/19312458.2012.679848

Preacher, K. J., Zyphur, M. J., \& Zhang, Z. (2010). A general multilevel SEM framework for assessing multilevel mediation. Psychological Methods, 15, 209-233. doi: 10.1037/a0020141

Rea, L. M., \& Parker, R. A. (1997). Designing and Conducting Survey Research: A Comprehensive Guide. San Francisco, CA: Josey-Bass Publishers.
Rafferty, A.E., \& Griffin, M. A. (2006). Perceptions of organizational change: A stress and coping perspective. Journal of Applied Psychology, 91(5), 1154-1162. doi: 10.1037/0021-9010.91.5.1154

Rafferty, A.E., \& Restubog, S. L. D. (2010). The impact of change process and context on change reactions and turnover during a merger. Journal of Management, 36, 1309-1338. doi: 10.1177\%2F0149206309341480

Self, D., Armenakis, A., \& Schraeder, M. (2007). Organizational change content, process and context: A simultaneous analysis of employee reactions. Journal of Change Management, 7(2), 211-229. doi: 10.1080/14697010701461129

Shoss, M. K., Witt, L.A., \& Vera, D. (2011). When does adaptive performance lead to higher task performance? Journal of Organizational Behavior, 33(7), 910-924. doi: 10.1002/job.780

Tabachnick, B. G., \& Fidell, L. S. (2013). Using multivariate statistics (6 ed.). Boston: Pearson International Education.

Vakola, M. (2016). The reasons behind change recipients' behavioral reactions: a longitudinal investigation. Journal of Managerial Psychology, 31(1), 202-215. doi: 10.1108/JMP-02-2013-0058. doi: 10.1108/JMP-02-2013-0058

Vakola, M.; Armenakis, A., \& Oreg, S. (2013). Reactions to organizational change from an individual differences perspective: A review of empirical research (95-122). In S. Oreg, A. Mitchel, \& R. Todnem By (Orgs.), The psychology of organizational change: Viewing change from the employee's perspective (pp.95-122). Cambridge: Cambridge University Press. doi: 10.1017/CBO9781139096690.008

Vakola, M., Tsaousis, L., \& Nikolaou, L. (2004). The role of emotional intelligence and personality variables on attitudes toward organizational change. Journal of Managerial Psychology, 19(2), 88-110. doi: 10.1108/02683940410526082

Valley, K. L., \& Thompson, T. A. (1998). Sticky ties and bad attitudes: relational and individual bases of resistance to change in organizational structure. In R. M. Kramer \& M. A. Neale (Orgs.), Power and influence in organizations. Londres: Sage publications, Inc.

Van de Ven, A. H., \& Sun, K. (2011). Breakdowns in implementing models of organization change. The Academy of Management Perspectives, 25(3), 58-74. doi: 10.5465/AMP.2011.63886530.

Vanessa Nery, Doutora em Psicologia Social, do Trabalho e das Organizações pela Universidade de Brasília - UnB, é gerente do Departamento de Projetos e Processos Corporativos da

Eletronorte. Endereço para correspondência: SQN 214 bloco A, apto 211, Asa Norte, Brasília DF. CEP 70.873-010.

E-mail: vanessa.nery@gmail.com

Elaine Rabelo Neiva, Doutora em Psicologia pela Universidade de Brasília - UnB, Pós-doutora em Psicologia Social e das Organizações na Columbia University em Nova Iorque, é professora na Universidade de Brasília no Programa de Pós-graduação em Psicologia Social, do Trabalho e das Organizações. E-mail: elaine_neiva@uol.com.br

Recebido em 10.jan.2017 Revisado em 10.fev.2019 Aceito em 03.jun.2019

Estudos de Psicologia, 23(4), outubro a dezembro de 2018, 369-380 